\title{
Back to the Lab? We Want to Hear From You
}

\section{As facilities open up, how are you finding the return to work?}

$\checkmark$ cientists around the world are slowly returning to their offices and labs. If you're one of them-or you're hoping to be soon-APS News and Physics want to hear about your experiences. What plans have you made to keep yourself or your group safe? How is distancing affecting your interactions? How have months away from the lab affected your perspective?

Tell us your story in a brief (100-200 words) letter to physics@aps.org. Pictures are welcome too. Each week, APS News and Physics will select a few of these letters and photos to share online.

Stay safe and healthy.

-The APS News and Physics Editorial Teams
Letters may be edited for brevity and clarity.

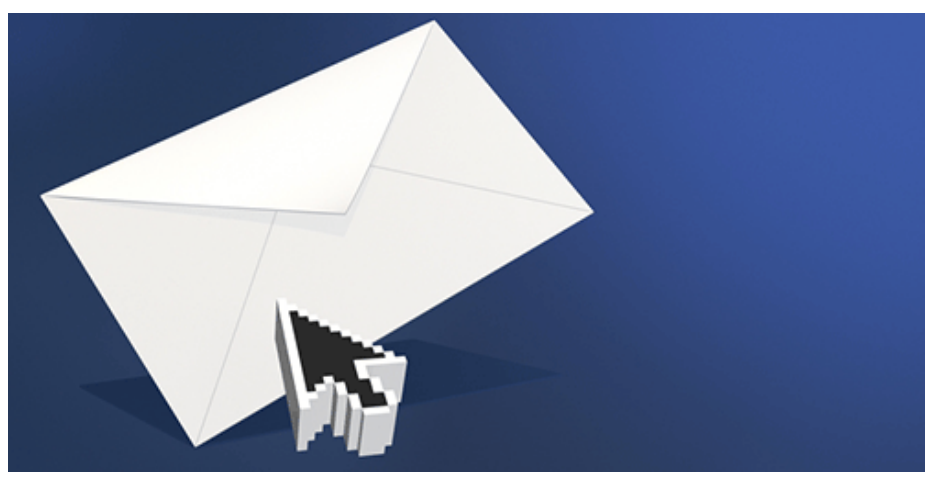

Credit: DSGpro/iStock/Getty Images 